Disclosure of interest statement No grants were received in the development of this study.

\section{P16.06 KNOWLEDGE OF KAPOSI SARCOMA (KS) AMONG HIV + COMMERCIAL SEX WORKERS ON HIV THERAPY IN KISUMU TOWNSHIP, KENYA}

C Kiplagat*, M Mutai, E Sumukwa. Jaramogi Oginga Odinga Teaching Hospital in Kisumu, Kenya

\subsection{6/sextrans-2015-052270.553}

Introduction The most common type of KS in the United States is epidemic or AIDS-related KS. Kaposi Sarcoma is a type of malignancy which develops in people who are infected with HIV, the virus that causes AIDS. Studies have suggested that the prevalence rate of KS is low on HIV Positive Persons due to an excellent ART therapy and remains higher on HIV Negative Persons.

Methods We performed a retrospective analysis in all HIV infected patients having KS using Demographic and Clinical parameters. We used outpatient Clinic at Jaramogi Oginga Odinga Teaching and referral Hospital in Kisumu Township as our main hospital for this research between the years of 2009-2014 respectively.

Results Among 2280 patients, 30 were diagnosed with KS in the period of 2009-2014. All Patients were commercial sex workers with a mean age of 35 (30-38). Kaposi Sarcoma was Diagnosed in different stages of HIV infection by using Cohorts.

Cohort 1: KS concomitant Diagnosis of HIV infection were 6 cases

Cohort II: Diagnosis of KS in cART naïve persons were 7 cases

Cohort III: Diagnosis of KS in Patients for the first 6 months of start of cART as IRIS were 4 cases

Conclusion All patients who were treated with 2NRTI+PI were permanently cured of KS. Also there was no KS cases observed on the stable and virological effective cART.

\section{P16.07 PRIOR CONDYLOMA AND SYPHILIS DIAGNOSIS AMONG HIV-INFECTED PERSONS: A NATIONWIDE POPULATION- BASED STUDY IN TAIWAN, 2000-2010}

${ }^{1} \mathrm{C}$ Strong ${ }^{*},{ }^{2} \mathrm{H}$ Zou, ${ }^{3} \mathrm{Y}$ Chen, ${ }^{1} \mathrm{C} \mathrm{Li}, 4,5 \mathrm{~W}$ Ko, ${ }^{4,6} \mathrm{~N} \mathrm{Ko}$. 'Department of Public Health, National Cheng Kung University, Tainan, 701, Taiwan; ${ }^{2}$ Kirby Institute, University of New South Wales, Sydney, 2052, Australia; ${ }^{3}$ Institute of Allied Health Sciences, College of Medicine, National Cheng Kung University, Tainan, 701, Taiwan; ${ }^{4}$ Department of Internal Medicine, National Cheng Kung University Hospital, Tainan, 701, Taiwan; ${ }^{5}$ College of Medicine, National Cheng Kung University, Tainan, 701, Taiwan; ${ }^{6}$ Department of Nursing, National Cheng Kung University, Tainan, 701, Taiwan

\subsection{6/sextrans-2015-052270.554}

Introduction Previously acquired sexually transmitted infections (STIs) increase the risk of HIV infection, yet few studies have examined the time interval between prior condyloma/syphilis diagnosis and HIV infection. We aimed to examine numbers of condyloma/syphilis diagnosis, and HIV screening prior to HIV infection in relation to HIV risk among HIV-infected persons. Methods This population-based study retrieved medical claims data from Taiwan National Health Insurance Research Database (NHIRD) from 2000 to 2010 and included 16119 HIV-infected persons more than 15 years of age. We included cases with prior condyloma/syphilis diagnosis before HIV infection and described the elapsed time between first condyloma/syphilis in the database and HIV infection. A subgroup analysis was conducted for those who have prior condyloma/syphilis diagnosis, using multivariable Cox proportional hazard regression to examine factors associated with HIV infection within 1 year.

Results One hundred and fifty-two HIV-infected persons received condyloma diagnosis prior to HIV infection with an average elapsed time of 644 days. Among them, 31\% became HIV-infected within one year after first diagnosis of condyloma in the database. For syphilis, 833 persons received syphilis diagnosis and the average elapsed time was 1025 days. Among them, $24 \%$ became HIV-infected within one year. For those who have been diagnosed with condyloma, each increment of condyloma diagnosis before HIV infection was associated with an 2.8 times of risk for HIV infection within 1 year $(95 \%$ confidence interval $(\mathrm{CI})=1.26-6.19)$. For those who were diagnosed with syphilis, numbers of condyloma and syphilis diagnosis and HIV screening were significantly associated with increased risk of HIV infection within 1 year, with adjusted hazard ratios (aHRs) of 3.97 (1.699.32), 1.95 (1.31-2.89), 1.72 (1.53-1.93), respectively.

Conclusion History of condyloma/syphilis before HIV infection was associated with risk of HIV infection among HIV-infected persons. More regular HIV screening among persons with STIs are warranted.

Disclosure of interest statement The authors declare that there are no conflicts of interest.

\section{P16.08 PREVALENCE OF SEXUALLY TRANSMITTED INFECTIONS AMONG PREGNANT HIV-POSITIVE IN CENTRAL-WEST BRAZIL}

SM Brunini ${ }^{1 *}$, J Valadares', LCC Guimarães ${ }^{2}$, BL Almeida2, CM Souza $2 .{ }^{1}$ Federal University of Goias, Brazil; ${ }^{2}$ Postgraduate Nursing Program of Federal University of Goias, Brazil

\subsection{6/sextrans-2015-052270.555}

Introduction In Brazil, the feminization of Human Immunodeficiency Virus epidemic (HIV) is a reality. This situation illustrates the occurrence of a period in a woman's life, pregnancy. In the HIV-positive co-infections are frequent, given that the virus shares the same transmission routes with other pathogens. In pregnant women, this condition is extremely worrying to consider the possibility of vertical transmission of HIV and other Sexually Trasmitted Infections (STIs) during pregnancy, childbirth or breastfeeding.

Methods A cross-sectional study, retrospective. Data collection was obtained from medical records. We reported the prevalence of other STIs in pregnant women living with HIV, met in the reference maternity of State Goias, Brazil, from January 2007 to December 2013.

Results Were evaluated 323 pregnant HIV-positive. Most women $(47,2 \%)$ were between 25 and 34 years of age, poorly educated $(74.2 \%)$ and reported being a housewife (75,3\%). 281 women $(79,6 \%)$ reported inconsistent condom use.

The overall prevalence of STDs was 14.5\% (95\% CI 11.02 18.72). Syphilis was detected in 5.0\% (95\% CI 2.96-7.75); Condylomatosis in $3.71 \%$ (95\% CI 2.13-6.38); Trichomonas vaginalis in $0.61 \%$ (95\% CI: $0.17-2.23)$. Serological markers of hepatitis B virus infection was present in 1.24\% (95\% CI: $0.48-$ 3.14). Vaginosis was also identified in $2.17 \%$ (95\% CI: $2.17-$ 4.40).

Conclusion The substancial prevalence of STIs including syphilis was found among pregnant women HIV-positive in the study. 